physics/9904063

\title{
Quantum aspects of accelerator optics *
}

\author{
Sameen Ahmed KHAN \\ Dipartimento di Fisica Galileo Galilei Università di Padova \\ Istituto Nazionale di Fisica Nucleare (INFN) Sezione di Padova \\ Via Marzolo 8 Padova 35131 ITALY \\ E-mail: khan@pd.infn.it, http://www.pd.infn.it/ khan/
}

\begin{abstract}
Present understanding of accelerator optics is based mainly on classical mechanics and electrodynamics. In recent years quantum theory of charged-particle beam optics has been under development. In this paper the newly developed formalism is outlined.
\end{abstract}

Keywords: Beam physics, Beam optics, Accelerator optics, Spin- $\frac{1}{2}$ particle, Anomalous magnetic moment, Quantum mechanics, Dirac equation, FoldyWouthuysen transformation, Polarization, Thomas-Bargmann-Michel-Telegdi equation, Magnetic quadrupole lenses, Stern-Gerlach kicks, Quantum corrections to the classical theory.

PACS: 29.20.-c (Cyclic accelerators and storage rings), 29.27.-a (Beams in particle accelerators), 29.27.Hj (Polarized beams), 41.75.-i (Charged-particle beams), 41.75.Ht (Relativistic electron and positron beams), 41.85.-p (Beam optics), 41.85.Ja (Beam transport), 41.85.Lc (Beam focusing and bending magnets).

*In Proceedings of the 1999 Particle Accelerator Conference (PAC99) 29 March - 02 April 1999, New York City, Editors: A. Luccio and W. MacKay 
Charged-particle beam optics, or the theory of transport of charged-particle beams through electromagnetic systems, is traditionally dealt with using classical mechanics. This is the case in ion optics, electron microscopy, accelerator physics etc [1]-沟. The classical treatment of charged-particle beam optics has been extremely successful, in the designing and working of numerous optical devices from electron microscopes to very large particle accelerators, including polarized beam accelerators. It is natural to look for a prescription based on the quantum theory, since any physical system is quantum at the fundamental level! Such a prescription is sure to explain the grand success of the classical theories and may also help towards a deeper understanding and designing of certain charged-particle beam devices. To date the curiosity to justify the success of the classical theories as a limit of a quantum theory has been the main motivation to look for a quantum prescription. But, with ever increasing demand for higher luminosities and the need for polarized beam accelerators in basic physics, we strongly believe that the quantum theories, which up till now were an isolated academic curiosity will have a significant role to play in designing and working of such devices.

It is historically very curious that the, quantum approaches to the chargedparticle beam optics have been very modest and have a very brief history as pointed out in the third volume of the three-volume encyclopaedic text book of Hawkes and Kasper [5]. In the context of accelerator physics the grand success of the classical theories originates from the fact that the de Broglie wavelength of the (high energy) beam particle is very small compared to the typical apertures of the cavities in accelerators. This and related details have been pointed out in the recent article of Chen [6. A detailed account of the quantum aspects of beam physics is to be found in the Proceedings of the recently held 15th Advanced ICFA Beam Dynamics Workshop [7].

A beginning of a quantum formalism starting ab initio with the Dirac equation was made only recently[8]-[9]. The formalism of Jagannathanet al was the first one to use the Dirac equation to derive the focusing theory of electron lenses, in particular for magnetic and electrostatic axially symmetric and quadrupole lenses respectively. This formalism further outlined the recipe to obtain a quantum theory of aberrations. Details of these and some of the related developments in the quantum theory of charged-particle beam optics can be found in the references [8]-[17]. I shall briefly state the central theme of the quantum formalism.

The starting point to obtain a quantum prescription is to build a theory based on the basic equations of quantum mechanics appropriate to the situation under study. For situations when either there is no spin or spinor effects are believed to be small and ignorable we start with the scalar Klein-Gordon and Schrödinger equations for relativistic and nonrelativistic cases respectively. For electrons, protons and other spin- $\frac{1}{2}$ particles it is natural to start with the Dirac equation, the equation for spin- $\frac{1}{2}$ particles. In practice we do not have to care about the other (higher spin) equations. 
In many situations the electromagnetic fields are static or can reasonably assumed to be static. In many such devices one can further ignore the times of flights which are negligible or of not direct interest as the emphasis is more on the profiles of the trajectories. The idea is to analyze the evolution of the beam parameters of the various individual charged-particle beam optical elements (quadrupoles, bending magnets, ...) along the optic axis of the system. This in the language of the quantum formalism would require to know the evolution of the wavefunction of the beam particles as a function of ' $s$ ', the coordinate along the optic axis. Irrespective of the starting basic time-dependent equation (Schrödinger, Klein-Gordon, Dirac, $\cdots$ ) the first step is to obtain an equation of the form

$$
\mathrm{i} \hbar \frac{\partial}{\partial s} \psi(x, y ; s)=\hat{\mathcal{H}}(x, y ; s) \psi(x, y ; s),
$$

where $(x, y ; s)$ constitute a curvilinear coordinate system, adapted to the geometry of the system. For systems with straight optic axis, as it is customary we shall choose the optic axis to lie along the $Z$-axis and consequently we have $s=z$ and $(x, y ; z)$ constitutes a rectilinear coordinate system. Eq. (11) is the basic equation in the quantum formalism and we call it as the beam-optical equation; $\mathcal{H}$ and $\psi$ as the beam-optical Hamiltonian and the beam wavefunction respectively. The second step requires to obtain a relationship for any relevant observable $\{\langle O\rangle(s)\}$ at the transverse-plane at $s$ to the observable $\left\{\langle O\rangle\left(s_{\text {in }}\right)\right\}$ at the transverse plane at $s_{\text {in }}$, where $s_{\text {in }}$ is some input reference point. This is achieved by the integration of the beam-optical equation in (1)

$$
\psi(x, y ; s)=\hat{U}\left(s, s_{\text {in }}\right) \psi\left(x, y ; s_{\text {in }}\right),
$$

which gives the required transfer maps

$$
\begin{aligned}
\langle O\rangle\left(s_{\text {in }}\right) & \rightarrow\langle O\rangle(s) \\
& =\langle\psi(x, y ; s)|O| \psi(x, y ; s)\rangle \\
& =\left\langle\psi\left(x, y ; s_{\text {in }}\right)\left|\hat{U}^{\dagger} O \hat{U}\right| \psi\left(x, y ; s_{\text {in }}\right)\right\rangle .
\end{aligned}
$$

The two-step algorithm stated above may give an over-simplified picture of the quantum formalism than, it actually is. There are several crucial points to be noted. The first-step in the algorithm of obtaining the beam-optical equation is not to be treated as a mere transformation which eliminates $t$ in preference to a variable $s$ along the optic axis. A clever set of transforms are required which not only eliminate the variable $t$ in preference to $s$ but also gives us the $s$-dependent equation which has a close physical and mathematical analogy with the original $t$-dependent equation of standard time-dependent quantum mechanics. The imposition of this stringent requirement on the construction of the beam-optical equation ensures the execution of the second-step of the algorithm. The beam-optical equation is such, that all the required rich machinery 
of quantum mechanics becomes applicable to compute the transfer maps characterizing the optical system. This describes the essential scheme of obtaining the quantum formalism. Rest is mostly a mathematical detail which is built in the powerful algebraic machinery of the algorithm, accompanied with some reasonable assumptions and approximations dictated by the physical considerations. For instance, a straight optic axis is a reasonable assumption and paraxial approximation constitute a justifiable approximation to describe the ideal behaviour.

Before explicitly looking at the execution of the algorithm leading to the quantum formalism in the spinor case, we further make note of certain other features. Step-one of the algorithm is achieved by a set of clever transformations and an exact expression for the beam-optical Hamiltonian is obtained in the case of Schrödinger, Klein-Gordon and Dirac equations respectively, without resorting to any approximations! We expect this to be true even in the case of higher-spin equations. The approximations are made only at step-two of the algorithm, while integrating the beam-optical equation and computing the transfer maps for averages of the beam parameters. Existence of approximations in the description of nonlinear behaviour is not uncommon and should come as no surprise, afterall the beam optics constitutes a nonlinear system. The nature of these approximations can be best summarized in the optical terminology as; a systematic procedure of expanding the beam optical Hamiltonian in a power series of $\left|\hat{\boldsymbol{\pi}}_{\perp} / p_{0}\right|$ where $p_{0}$ is the design (or average) momentum of beam particles moving predominantly along the direction of the optic axis and $\hat{\boldsymbol{\pi}}_{\perp}$ is the small transverse kinetic momentum. The leading order approximation along with $\left|\hat{\boldsymbol{\pi}}_{\perp} / p_{0}\right| \ll 1$ constitutes the paraxial or ideal behaviour and higher order terms in the expansion give rise to the nonlinear or aberrating behaviour. It is seen that the paraxial and aberrating behaviour get modified by the quantum contributions which are in powers of the de Broglie wavelength $\left(\lambda_{0}=2 \pi \hbar / p_{0}\right)$. Lastly, and importantly the question of the classical limit of the quantum formalism; it reproduces the well known Lie algebraic formalism of charged-particle beam optics pioneered by Dragt et al $[18]$.

We start with the Dirac equation in the presence of static electromagnetic field with potentials $(\phi(\boldsymbol{r}), \boldsymbol{A}(\boldsymbol{r}))$

$$
\hat{\mathrm{H}}_{D}\left|\psi_{D}\right\rangle=E\left|\psi_{D}\right\rangle,
$$

where $\left|\psi_{D}\right\rangle$ is the time-independent 4-component Dirac spinor, $E$ is the energy of the beam particle and the Hamiltonian $\hat{\mathrm{H}}_{D}$, including the Pauli term in the usual notation is

$$
\hat{\mathrm{H}}_{D}=\beta m_{0} c^{2}+c \boldsymbol{\alpha} \cdot \hat{\boldsymbol{p}}-\mu_{a} \beta \boldsymbol{\Sigma} \cdot \boldsymbol{B},
$$

where $\hat{\boldsymbol{\pi}}=\hat{\boldsymbol{p}}-q \boldsymbol{A}=-i \hbar \boldsymbol{\nabla}-q \boldsymbol{A}$. After a series of transformations (see [14] for details) we obtain the accelerator optical Hamiltonian to the leading order 
approximation

$$
\begin{aligned}
\mathrm{i} \hbar \frac{\partial}{\partial z}\left|\psi^{(A)}\right\rangle= & \hat{H}^{(A)}\left|\psi^{(A)}\right\rangle \\
\hat{H}^{(A)} \approx & \left(-p_{0}-q A_{z}+\frac{1}{2 p_{0}} \hat{\pi}_{\perp}^{2}\right) \\
& +\frac{\gamma m_{0}}{p_{0}} \underline{\boldsymbol{\Omega}}_{s} \cdot \boldsymbol{S} \\
\text { with } \underline{\boldsymbol{\Omega}}_{s}= & -\frac{1}{\gamma m_{0}}\left\{q \boldsymbol{B}+\epsilon\left(\boldsymbol{B}_{\|}+\gamma \boldsymbol{B}_{\perp}\right)\right\}
\end{aligned}
$$

where $\hat{\pi}_{\perp}^{2}=\hat{\pi}_{x}^{2}+\hat{\pi}_{y}^{2}, \epsilon=2 m_{0} \mu_{a} / \hbar, \gamma=E / m_{0} c^{2}$, and $\boldsymbol{S}=\frac{1}{2} \hbar \boldsymbol{\sigma}$. We can recognize $\hat{H}^{(A)}$ as the quantum mechanical, accelerator optical, version of the well known semiclassical Derbenev-Kondratenko Hamiltonian [19] in the leading order approximation. We can obtain corrections to this by going an order beyond the first order calculation.

It is straightforwrd to compute the transfer maps for a specific geometry and the detailed discussion with the quantum corrections can be found in [14]. In the classical limit we recover the Lie algebraic formalism [18.

One practical application of the quantum formalism would be to get a deeper understanding of the polarized beams. A proposal to produce polarized beams using the proposed spin-splitter devices based on the classical Stern-Gerlach kicks has been presented recently [20].

Lastly it is speculated that the quantum theory of charged-particle beam optics will be able to resolve the choice of the position operator in the Dirac theory and the related question of the form of the force experienced by a charged-particle in external electromagnetic fields [19], 21]. This will be possible provided one can do an extremely high precision experiment to detect the small differences arising in the transfer maps from the different choices of the position operators. These differences shall be very small, i.e., proportional to powers of the de Broglie wavelength. It is the extremely small magnitude of these minute differences which makes the exercise so challenging and speculative!

\section{References}

[1] P.W. Hawkes and E. Kasper, Principles of Electron Optics, Vols. I and II (Academic Press, London, 1989).

[2] M. Conte and W.W. MacKay, An Introduction to the Physics of Particle Accelerators (World Scientific, Singapore, 1991).

[3] H. Mais, "Some topics in beam dynamics of storage rings", DESY 96-119 (1996). 
[4] H. Wiedemann, Particle Accelerator Physics : Basic Principles and Linear Beam Dynamics (Springer-Verlag, Berlin, Heidelberg, 1993)

H. Wiedemann, Particle Accelerator Physics II : Nonlinear and HigherOrder Beam Dynamics (Springer-Verlag, Berlin, Heidelberg, 1995)

[5] P.W. Hawkes and E. Kasper, Principles of Electron Optics Vol.3: Wave Optics (Academic Press, London and San Diego, 1994).

[6] P. Chen, ICFA Beam Dynamics Newsletter 12, 46 (1996);

[7] Proceedings of the 15th Advanced ICFA Beam Dynamics Workshop on Quantum Aspects of beam Physics, Ed. P. Chen, (World Scientific, Singapore, 1999).

[8] R. Jagannathan, R. Simon, E. C. G. Sudarshan and N. Mukunda, Phys. Lett. A 134, 457 (1989); R. Jagannathan, in Dirac and Feynman: Pioneers in Quantum Mechanics, Ed. R. Dutt and A. K. Ray (Wiley Eastern, New Delhi, 1993).

[9] R. Jagannathan, Phys. Rev. A 42, 6674 (1990).

[10] S.A. Khan and R. Jagannathan, "Theory of relativistic electron beam transport based on the Dirac equation", Proc. of the 3rd National Seminar on Physics and Technology of Particle Accelerators and their Applications, (Nov. 1993, Calcutta, India) Ed. S. N. Chintalapudi (IUC-DAEF, Calcutta) 102; S.A. Khan and R. Jagannathan, "Quantum mechanics of chargedparticle beam optics: An operator approach", Presented at the JSPS-KEK International Spring School on High Energy Ion Beams-Novel Beam Techniques and their Applications, March 1994, Japan, Preprint: IMSc/94/11 (The Institute of Mathematical Sciences, Madras, March 1994).

[11] S.A. Khan and R. Jagannathan, Phys. Rev. E 51, 2510 (1995).

[12] R. Jagannathan and S.A. Khan, Advances in Imaging and Electron Physics, 97, Ed. P. W. Hawkes (Academic Press, San Diego) 257 (1996).

[13] S.A. Khan, Quantum Theory of Charged-Particle Beam Optics, Ph.D. Thesis (University of Madras) (1997).

[14] M. Conte, R. Jagannathan, S.A. Khan and M. Pusterla, Part. Accel. 56, 99 (1996).

[15] R. Jagannathan and S.A. Khan ICFA Beam Dynamics Newsletter 13, 21 (1997).

[16] R. Jagannathan, "The Dirac equation approach to spin- $\frac{1}{2}$ particle beam optics", in: Proceedings of the 15th Advanced ICFA Beam Dynamics Workshop on Quantum Aspects of beam Physics, Ed. P. Chen, (World Scientific, Singapore, 1999), physics $/ 9803042$. 
[17] S. A. Khan, Quantum theory of magnetic quadrupole lenses for spin- $\frac{1}{2}$ particles, in: Proceedings of the 15th Advanced ICFA Beam Dynamics Workshop on Quantum Aspects of beam Physics, Ed. P. Chen, (World Scientific, Singapore, 1999), physics/9809032.

[18] A.J. Dragt and E. Forest, Adv. Electronics and Electron Phys. 67, 65 (1986); A.J. Dragt, F. Neri, G. Rangarajan, D.R. Douglas, L.M. Healy and R.D. Ryne, Ann. Rev. Nucl. Part. Sci. 38, 455 (1988); G. Rangarajan, A.J. Dragt and F. Neri, Part. Accel. 28, 119 (1990); R.D. Ryne and A.J. Dragt, Part. Accel. 35, 129 (1991); É. Forest and K. Hirata, A Contemporary Guide to Beam Dynamics KEK Report 92-12; É. Forest, M. Berz and J. Irwin, Part. Accel. 24, 91 (1989); K. Yokoya, "Calculation of the equilibrium polarization of stored electron beams using Lie algebra", Preprint KEK 86-90 (1986); Yu.I. Eidelman and V.Ye. Yakimenko, Part. Accel. 45, 17 (1994); and references therein.

[19] "On Stern-Gerlach forces allowed by special relativity and the special case of the classical spinning particle of Derbenev-Kondratenko", e-print: physics/9611001; D. P. Barber, K. Heinemann and G. Ripken, Z. Phys. C 64 (1994) 117; D. P. Barber, K. Heinemann and G. Ripken, Z. Phys. C 64 (1994) 143.

[20] M. Conte, A. Penzo and M. Pusterla, Il Nuovo Cimento A 108, 127 (1995); Y. Onel, A. Penzo and R. Rossmanith, AIP Conf. Proc. 150 Ed. R. G. Lernerand and D. F. Geesaman, (AIP, New York, 1986) 1229; M. Conte, and M. Pusterla, Il Nuovo Cimento A, 103, 1087 (1990); M. Conte, Y. Onel, A. Penzo, A. Pisent, M. Pusterla and R. Rossmanith, The spin-splitter concept, Internal Report : INFN/TC-93/04; M. Pusterla, "Polarized beams and Stern-Gerlach forces in classical and quantum mechanics", in: Proceedings of the 15th Advanced ICFA Beam Dynamics Workshop on Quantum Aspects of beam Physics, Ed. P. Chen, (World Scientific, Singapore, 1999),

[21] A. O. Barut and R. Raczka, Theory of Group Representations and Applications (World Scientific, 1986); J. Anandan, Nature 387, 558 (1997); M. Chaichian. R. G. Felipe and D. L. Martinez, Phys. Lett. A 236, 188 (1997); J. P. Costella and B. H. J. McKellar, Int. J. Mod. Phys. A 9, 461 (1994); and references therein. 